\title{
Biosimilar G-CSF Based Mobilization of Peripheral Blood Hematopoietic Stem Cells for Autologous and Allogeneic Stem Cell Transplantation
}

\author{
Michael Schmitt ${ }^{\circledR}$, Amy Publicover ${ }^{2}$, Kim H Orchard ${ }^{2}$, Matthias Görlach ${ }^{3}$, Lei Wang1, Anita Schmitt ${ }^{1}$, Jiju \\ Mani $^{1}$, Panagiotis Tsirigotis ${ }^{4}$, Reeba Kuriakose ${ }^{1}$, Arnon Nagler ${ }^{5}$ \\ 1. University Clinic Heidelberg, Internal Medicine V (Hematology, Oncology and Rheumatology) University of Heidelberg, 69120 Hei- \\ delberg, Germany. \\ 2. University Hospital Southampton, Southampton, United Kingdom. \\ 3. Research Group Biomolecular NMR Spectroscopy, Leibniz Institute for Age Research - Fritz Lipmann Institute e.V. (FLI), Jena, Germany \\ 4. 2nd Department of Internal Medicine, Propaedeutic, Attikon General University Hospital, University of Athens, Greece. \\ 5. Hematology Division, BMT and Cord Blood Bank, Tel-Aviv University, Chaim Sheba Medical Center, Tel-Hashomer, Israel.
}

$\triangle$ Corresponding author: Univ.-Prof. Dr. med. Michael Schmitt, MHBA, Head of Cellular Immunotherapy, Internal Medicine V, University Clinic Heidelberg, Im Neuenheimer Feld 410, 69120 Heidelberg. Tel. +49-6221-56-6614; FAX +49-6221-56-5740; michael.schmitt@med.uni-heidelberg.de

(c) Ivyspring International Publisher. This is an open-access article distributed under the terms of the Creative Commons License (http://creativecommons.org/ licenses/by-nc-nd/3.0/). Reproduction is permitted for personal, noncommercial use, provided that the article is in whole, unmodified, and properly cited.

Received: 2013.09.25; Accepted: 2013.12.29; Published: 2014.01 .23

\begin{abstract}
The use of granulocyte colony stimulating factor (G-CSF) biosimilars for peripheral blood hematopoietic stem cell (PBSC) mobilization has stimulated an ongoing debate regarding their efficacy and safety. However, the use of biosimilar G-CSF was approved by the European Medicines Agency (EMA) for all the registered indications of the originator G-CSF (Neupogen ${ }^{\circledR}$ ) including mobilization of stem cells. Here, we performed a comprehensive review of published reports on the use of biosimilar G-CSF covering patients with hematological malignancies as well as healthy donors that underwent stem cell mobilization at multiple centers using site-specific non-randomized regimens with a biosimilar G-CSF in the autologous and allogeneic setting.

A total of 904 patients mostly with hematological malignancies as well as healthy donors underwent successful autologous or allogeneic stem cell mobilization, respectively, using a biosimilar G-CSF (520 with Ratiograstim $® / T$ evagrastim, 384 with Zarzio $®$ ). The indication for stem cell mobilization in hematology patients included 326 patients with multiple myeloma, 273 with Non-Hodgkin's lymphoma (NHL), 79 with Hodgkin's lymphoma (HL), and other disease. 156 sibling or volunteer unrelated donors were mobilized using biosimilar G-CSF. Mobilization resulted in good mobilization of CD34+ stem cells with side effects similar to originator G-CSF. Post transplantation engraftment did not significantly differ from results previously documented with the originator G-CSF. The side effects experienced by the patients or donors mobilized by biosimilar G-CSF were minimal and were comparable to those of originator G-CSF.

In summary, the efficacy of biosimilar G-CSFs in terms of PBSC yield as well as their toxicity profile are equivalent to historical data with the reference G-CSF.
\end{abstract}

Key words: Biosimilar G-CSF, hematopoietic stem cells, mobilization, autologous \& allogeneic transplantation, healthy donors 


\section{Granulocyte colony stimulating factor (G-CSF)}

Granulocyte colony stimulating factor (G-CSF) has become a widely used clinical tool used by hematologists and oncologists to treat therapy-induced neutropenia and to accelerate and potentiate engraftment after hematopoietic stem cell transplantation. $[1,2]$

\section{Biosimilar G-CSF}

When conventional drugs produced by chemical synthesis contain the same active substance as the original agent and are similar in terms of quality, safety and efficacy to the original drug, they are termed 'generic'. Hematopoietic growth factors including G-CSF are manufactured by the use of recombinant technology and for regulatory purposes are classified as 'biological medicines' and as such, must comply with specific manufacturing requirements.[3] When biologically equivalent agents are manufactured they are termed "biosimilars". [4] The EMEA stated that "a company may choose to develop a new biological medicinal product claimed to be similar (Similar Biological Medicinal Product) in terms of Quality, Safety and Efficacy to an original, reference medicinal product, which has been granted a marketing authorization in the Community." [3] The manufacture and use of biosimilar G-CSF has a specific set of guidance notes produced by the EMEA, now referred to as EMA.[3] The use of biosimilars in general and in particular for peripheral blood hematopoietic stem cell (PBSC) mobilization has stimulated an ongoing debate regarding their efficacy and safety.[4-8]

\section{Extrapolation for biosimilars}

The use of biosimilar G-CSF (Ratiograstim $^{\circledR}$, Tevagrastim ${ }^{\circledR}$, Biograstim ${ }^{\circledR}$, Zarzio $^{\circledR}$, Nivestim $\left.{ }^{\circledR}\right)$ was approved by the European Medicines Agency (EMA) for all the registered indications of the originator $\left(\right.$ Neupogen $\left.{ }^{\circledR}\right)$ including chemotherapy induced neutropenia (CIN), agranulocytosis and neutropenia due to infection with the human immunodeficiency virus (HIV) and mobilization of stem cells in the autologous and allogeneic settings, based on their comparable efficacy and safety profile to the originator G-CSF in CIN.[9] Comparability of biosimilar G-CSF with the originator filgrastim was assessed in three large randomized two-arm comparative studies in a single indication for which the reference G-CSF is approved, i.e. the efficacy of originator versus biosimilar G-CSF in CIN in patients with breast cancer, lung cancer and malignant lymphoma.[10] The extrapolation from these positive results indicating an identical efficacy and safety profile when compared to originator filgrastim (Neupogen ${ }^{\circledR}$ ) to the use of the biosimilar G-CSF for the mobilization of CD34+ hematopoietic stem cells in healthy donors was based on European Law.[9] However, this extrapolation in general raised questions. $[3-5,7,11]$

\section{Similarity of structure and production process of originator and biosimilar G-CSF}

The World Health Organization (WHO) stated that "the clinical performance of biotherapeutics can also be much influenced by the manufacturing process and therefore some clinical studies will also be required to support the safety and efficacy of a similar biotherapeutic product (SBP)." [12]

Filgrastim, the active substance of Ratiograstim ${ }^{\circledR} /$ Tevagrastim $\AA$ is a non- glycosylated recombinant $\mathrm{N}$-methionyl human granulocyte colony stimulating factor expressed in E. coli and consisting of 175 amino acids (Figure 1). [13-15]

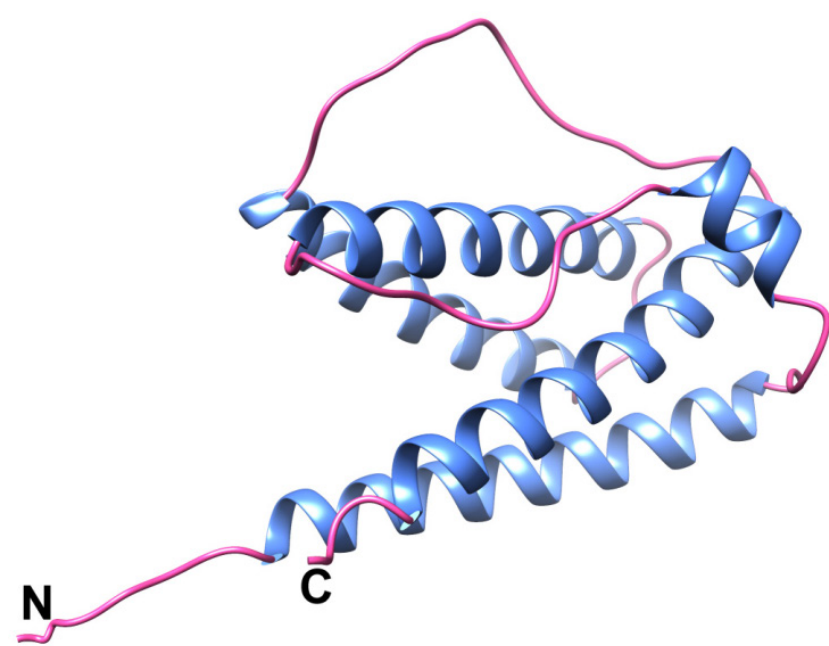

Figure I. Structure of recombinant human G-CSF. Ribbon representation of the structure of recombinantly produced human G-CSF. The sequence of this biosimilar is identical to the one of filgrastim (Neupogen $($ ). Helical regions are colored in blue, coil regions in pink. The $\mathrm{N}$ - and the $\mathrm{C}$-terminus are indicated. Structure co-ordinates (PDB IGNC; [15]) were used to calculate a mean structure with MOLMOL [13]. The ribbon representation was generated using the UCSF Chimera package [14].

The E. coli host strain was transformed with the plasmid using standard techniques to generate the recombinant strain E. coli for production of G-CSF. During the biosynthesis process, G-CSF protein is expressed in inclusion bodies in the cells. After a predefined growth time cells are harvested, disrupted and inclusion bodies washed by buffer for removal of contaminants. The inclusion bodies are dissolved in 
chaotropic agent and refolded in a reducing-oxidizing system. After refolding, a series of orthogonal chromatographic purification steps are applied. Following purification, the $\mathrm{XM} 02$ active substance is filtered and stored at 2 to $8^{\circ} \mathrm{C}$.

Ratiograstim $® /$ Tevagrastim ${ }^{\circledR}$ in a concentration of $0.6 \mathrm{mg} / \mathrm{ml}$ is supplied in two dosage strengths 30 $\mathrm{MIU} / 0.5 \mathrm{ml}$ and $48 \mathrm{MIU} / 0.8 \mathrm{ml}$, filled in $1 \mathrm{ml}$ glass, single-use, pre-filled syringes. The formulation of Ratiograstim ${ }^{\circledR} /$ Tevagrastim ${ }^{\circledR}$ has the same excipients as Neupogen ${ }^{\circledR}$, i.e. acetic acid, polysorbate 80 , sodium hydroxide, sorbitol and water for injections (as per product information).

As required for a similar biological medicinal product, comparability to the reference medicinal product Neupogen ${ }^{\circledR}$ has been demonstrated through an extensive head-to-head characterization and stability studies performed for both XM02 active substance and medicinal product. Physical properties, the primary and higher order structures, the biological activity and product related impurities, found to be similar to Neupogen ${ }^{\circledR}$ (data on file).

\section{Statements from WMDA and EBMT on the use of biosimilar G-CSF}

Currently, there are few published data regarding the use of biosimilar G-CSF in the context of autologous PBSC for mobilization. Almost no published data exist as for hematopoietic stem cell mobilization from healthy donors. In a recent review by Shaw et al., the World Marrow Donor Association (WMDA) recommends that "biosimilars should only be used in healthy donors where the donor is entered and followed on a clinical study." [16] In 2009, the Executive Committee of the European Bone Marrow Transplantation (EBMT) Association issued a letter stating that "until studies have been performed to provide the required efficacy and safety data, the EBMT does not recommend the use of biosimilar G-CSFs for mobilization of stem cells in healthy donors for stem cell transplantation." The recommendation from the EBMT Executive was that only after collection of comprehensive data G-CSF biosimilars could be considered routinely for the mobilization of peripheral blood stem cells in sibling and volunteer donors.[17]

\section{Assessment of the wide use of G-CSF in the real transplantation world}

We performed a comprehensive review of published reports covering 904 patients, with hematological malignancies as well as healthy donors that underwent stem cell mobilization with a biosimilar G-CSF for autologous and allogeneic stem cell transplantation, or cellular therapies for tissue regenera- tion, evaluating mobilization yield and safety profile as well as engraftment and transplantation outcome.

The database pubmed.org and the abstract books of the Annual Meetings of the European Bone Marrow Transplantation (EBMT) Association in 2012 and 2013 were reviewed for peer-reviewed papers and peer-reviewed abstracts respectively regarding the mobilization of stem cells with a biosimilar G-CSF.

An extensive literature review[18-39] produced 904 patients mostly with hematological malignancies as well as healthy donors that underwent successful autologous or allogeneic stem cell mobilization respectively using a biosimilar G-CSF (Ratiograstim ${ }^{\circledR} /$ Tevagrastim ${ }^{\circledR}$ or Zarzio ${ }^{\circledR}$ ). A total of 520 patients or donors underwent stem cell mobilization with Ratiograstim ${ }^{\circledR} /$ Tevagrastim $\AA$, while 384 patients or donors underwent stem cell mobilization with Zarzio®. The indication for stem cell mobilization in hematology patients included those with multiple myeloma, Non-Hodgkin lymphoma (NHL), Hodgkin's lymphoma (HL), acute and chronic leukemia. Patients with germ cell tumors as well as a small number of patients with cardiac failure who were in cell therapy studies were also included. Sibling or volunteer unrelated donors were mobilized using either Ratiograstim ${ }^{\circledR} /$ Tevagrastim ${ }^{\circledR}$ or Zarzioß. In Figure 2 the total number and the underlying disease of the patients that underwent autologous stem cell mobilization with Ratiograstim ${ }^{\circledR} /$ Tevagrastim ${ }^{\circledR}$ or Zarzio ${ }^{\circledR}$ is detailed, while Figure 3 summarizes the healthy donors that were mobilized with a biosimilar G-CSF respectively. The details of the specific biosimilar G-CSF used including dose, mode of autologous mobilization and indication are summarized in Table 1, while Table 2 shows the details of the equivalent procedure in healthy donors. It is of note that biosimilar G-CSF was already used for hematopoietic stem cell mobilization not just in sibling donors but also of unrelated volunteer donors from donor registries (Table 2). Mobilization parameters, yield, toxicity and post autologous or allogeneic transplantation engraftment data are detailed in Table 3 and Table 4 respectively. Biosimilar G-CSF based stem cell mobilization for both autologous and allogeneic transplantation resulted in good mobilization of CD34+ stem cells with side effects similar to reference G-CSF. Post transplantation engraftment did not significantly differ from results previously documented with the originator filgrastim $\left(\right.$ Neupogen $\left.{ }^{\circledR}\right)$ in historical controls. The side effects experienced by the patients or donors mobilized by biosimilar G-CSF (Ratiograstim ${ }^{\circledR}$ or Tevagrastim ${ }^{\circledR}$ or Zarzio $\left.{ }^{\circledR}\right)$ were minimal and were comparable to those of originator G-CSF (Table 3 and Table 4). 
We could not detect any difference in kinetics of mobilization of PBSC and graft composition between biosimilar and G-CSF. This applies to the cell counts for nucleated cells (NC) as well as to CD34+ progeni- tor cells, Natural Killer (NK) and T cells. Neither the kinetics nor the ratio of these cell subsets differed between biosimilar and originator G-CSF.

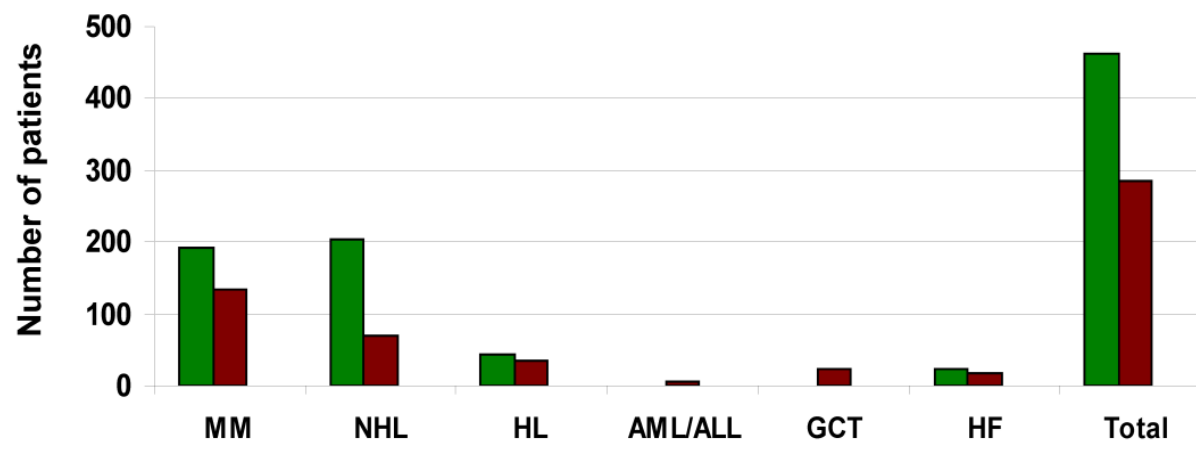

Figure 2. Patients undergoing autologous hematopoietic stem cell mobilization with Ratiograstim ${ }^{\circledR} /$ Tevagrastim ${ }^{\circledR}$ or Zarzio $\circledast$ (Sandoz). The y-axis shows the number of patients with multiple myeloma (MM), non-Hodgkin Lymphoma (NHL), Hodgkin's disease (HL), acute myeloid/lymphoblastic leukemia (AML/ALL), relapsed germinal cell tumor (GCT) or heart failure (HF) as well as the number of all patients (total) undergoing autologous hematopoietic stem cell mobilization with Ratiograstim ${ }^{\circledR} /$ Tevagrastim ${ }^{\circledR}$ [green bars] or Zarzio ${ }^{\circledR}$ (Sandoz) [brown bars].

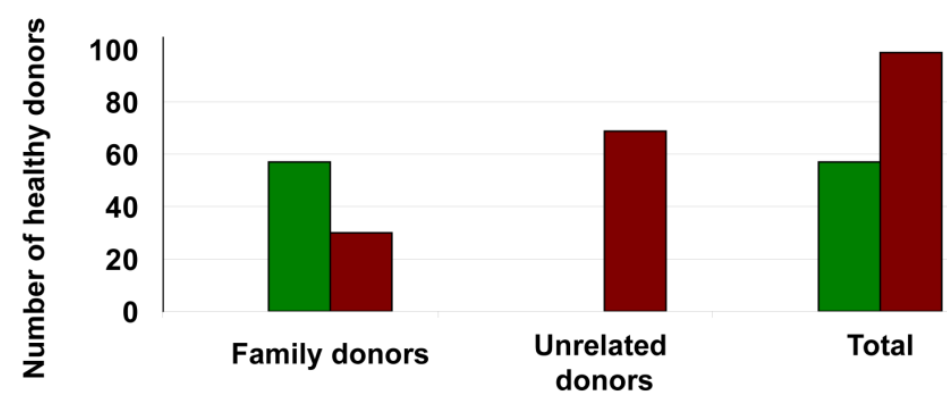

Figure 3. Healthy donors undergoing hematopoietic stem cell mobilization with Ratiograstim $\AA^{\circ}$ Tevagrastim ${ }^{\circledR}$ or Zarzio $($ (Sandoz) for allogeneic stem cell transplantation. The y-axis shows the number of donors, numbers for family donors vs. unrelated donors are specified as well as the number of all patients (total) undergoing hematopoietic stem cell mobilization with Ratiograstim $®$ / Tevagrastim $®$ [green bars] or Zarzio ${ }^{(S a n d o z}$ ) [brown bars] for allogeneic stem cell transplantation.

Table I. Biosimilar G-CSF - Mode and dose for autologous hematopoietic stem cell mobilization

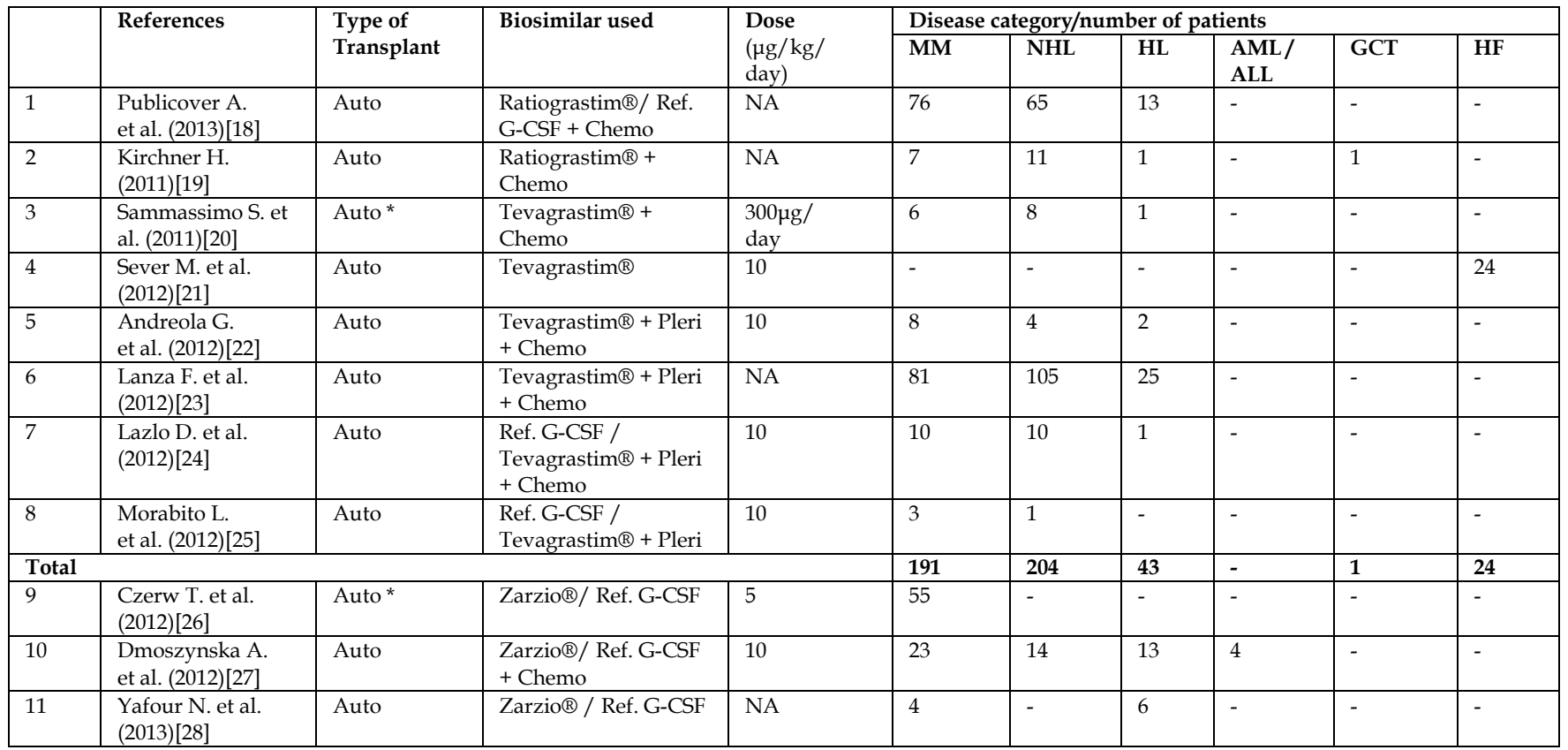




\begin{tabular}{|c|c|c|c|c|c|c|c|c|c|c|}
\hline & \multirow[t]{2}{*}{ References } & \multirow{2}{*}{$\begin{array}{l}\text { Type of } \\
\text { Transplant }\end{array}$} & \multirow[t]{2}{*}{ Biosimilar used } & \multirow{2}{*}{$\begin{array}{l}\text { Dose } \\
(\mu \mathrm{g} / \mathrm{kg} / \\
\text { day) }\end{array}$} & \multicolumn{6}{|c|}{ Disease category/number of patients } \\
\hline & & & & & MM & NHL & HL & $\begin{array}{l}\text { AML/ } \\
\text { ALL }\end{array}$ & GCT & HF \\
\hline 12 & $\begin{array}{l}\text { Kotwica K. et al. } \\
\text { (2012)[29] }\end{array}$ & Auto * & Zarzio $®$ + Chemo & NA & 12 & 4 & 6 & 1 & - & - \\
\hline 13 & $\begin{array}{l}\text { Gopcsa L. et al. } \\
(2013)[30]\end{array}$ & Auto & Zarzio $®$ + Chemo & NA & 11 & 8 & 2 & - & - & - \\
\hline 14 & $\begin{array}{l}\text { Ostuni A. et al. } \\
(2013)[31]\end{array}$ & Auto & Zarzio® + Chemo & 10 & 11 & 22 & 9 & $2\left(1+1^{* *}\right)$ & - & - \\
\hline 15 & $\begin{array}{l}\text { Sever M. et al. } \\
\text { (2013)[32] }\end{array}$ & Auto & Zarzio® & 10 & - & - & - & - & - & 16 \\
\hline 16 & $\begin{array}{l}\text { De Giorgi U. et al. } \\
\text { (2012)[33] }\end{array}$ & Auto & Zarzio® + Chemo & NA & - & - & - & - & 22 & - \\
\hline 17 & $\begin{array}{l}\text { Lefrere F. et.al. } \\
\text { (2011)[34] }\end{array}$ & Auto & Zarzio® + Chemo & $5-10$ & 19 & 21 & - & - & - & - \\
\hline \multicolumn{5}{|c|}{ Total } & 135 & 69 & 36 & 7 & 22 & 16 \\
\hline
\end{tabular}

Auto - Autologous mobilization; Auto*- Autologous transplantation; Pleri - Plerixafor; Ref. G-CSF - Reference G-CSF (Neupogen ${ }^{\circledR}$, Amgen); Chemo- Chemotherapy; MM Multiple Myeloma; NHL - Non Hodgkin Lymphoma; HL - Hodgkin's Lymphoma; AML / ALL - Acute Myeloid Leukemia / Acute Lymphoblastic Leukemia; ** - Acute Lymphoblastic Leukemia ; GCT- Relapsed Germ cell tumors; HF - Heart failure; NA - not available from abstract

Table 2. Biosimilar G-CSF for hematopoietic stem cell mobilization for allogeneic stem cell transplantation

\begin{tabular}{|c|c|c|c|c|c|c|}
\hline & \multirow{2}{*}{ References } & \multirow[t]{2}{*}{ Type of Transplant } & \multirow[t]{2}{*}{ Biosimilar used } & \multirow{2}{*}{$\begin{array}{l}\text { Dose } \\
(\mu \mathrm{g} / \mathrm{kg} / \text { day })\end{array}$} & \multicolumn{2}{|c|}{ Number of Healthy donors } \\
\hline & & & & & $\begin{array}{l}\text { Family Do- } \\
\text { nors }\end{array}$ & Unrelated donors \\
\hline 1 & Schmitt M. et al. (2013)[35] & Allogeneic & Ratiograstim ${ }^{\circledR / R e f . ~ G-C S F}$ & 20 & 11 & - \\
\hline 2 & $\begin{array}{l}\text { Nagler A. et al. (un- } \\
\text { published) }\end{array}$ & Allogeneic & Tevagrastim ${ }^{\circledR}$ & 24 & 22 & - \\
\hline 3 & Nagler A. et al. (2013)[36] & Allogeneic & Tevagrastim $\AA$ & 10 & 24 & - \\
\hline \multicolumn{5}{|c|}{ Total } & 57 & - \\
\hline 4 & $\begin{array}{l}\text { Antelo ML. et al. } \\
\text { (2013)[37] }\end{array}$ & Allogeneic & $\begin{array}{l}\text { Zarzio® / } \\
\text { Ref. G-CSF }\end{array}$ & 5 & 9 & - \\
\hline 5 & $\begin{array}{l}\text { Becker PSA. et al. } \\
(2013)[38]\end{array}$ & Allogeneic & Zarzio® & NA & - & 69 \\
\hline 6 & Azar N. Et al. (2012)[39] & Allogeneic & Zarzio® & 10 & 21 & - \\
\hline \multicolumn{5}{|c|}{ Total } & 30 & 69 \\
\hline
\end{tabular}

Ref. G-CSF - Reference G-CSF (Neupogen®, Amgen); NA - not available from abstract

Table 3. Mobilization and engraftment data of patients undergoing autologous hematopoietic stem cell mobilization with biosimilar G-CSF

\begin{tabular}{|c|c|c|c|c|c|c|c|c|c|}
\hline & \multirow[t]{3}{*}{ References } & \multirow{3}{*}{$\begin{array}{l}\text { No. of } \\
\text { pa- } \\
\text { tients }\end{array}$} & \multirow{3}{*}{$\begin{array}{l}\text { No. of } \\
\text { apheresis }\end{array}$} & \multirow{3}{*}{$\begin{array}{l}\text { CD34+ cell count } \\
\text { ( X 106/kg body } \\
\text { weight) }\end{array}$} & \multirow{3}{*}{$\begin{array}{l}\text { No. of CD34+ } \\
\text { cells/ } / \mu 1\end{array}$} & \multicolumn{3}{|c|}{ Engraftment (Time till regeneration) } & \multirow[t]{3}{*}{ Side Effects } \\
\hline & & & & & & \multirow{2}{*}{$\begin{array}{l}\begin{array}{l}\text { Neutro- } \\
\text { phils }\end{array} \\
>0.5 \mathrm{G} / \mathrm{L} \\
\end{array}$} & \multicolumn{2}{|c|}{ Platelets } & \\
\hline & & & & & & & $>20 \mathrm{G} / \mathrm{L}$ & $>50 \mathrm{G} / \mathrm{L}$ & \\
\hline 1 & $\begin{array}{l}\text { Publicover A. } \\
\text { et al. } \\
\text { (2013)[18] }\end{array}$ & 154 & $\begin{array}{l}66 \% \text { patients }=1 \\
28 \% \text { patients }=2 \\
6 \% \text { patients }=3 \\
1 \% \text { patients }=4 \\
{[\text { Mean }=1.4} \\
( \pm 1.2)]\end{array}$ & $\begin{array}{l}\text { Median= 4.53 } \\
\text { (range= } 0.2- \\
43.4) \\
{[\text { Mean= }} \\
6.6( \pm 13.8)]\end{array}$ & $\begin{array}{l}\text { Median= } 38 \\
(\text { range }= \\
0-516)\end{array}$ & $\begin{array}{l}\text { Median= } \\
13 \text { days } \\
\text { (range }=9- \\
22 \text { days) } \\
{[\text { Mean= }} \\
13( \pm 6) \\
\text { days }]\end{array}$ & $\begin{array}{l}\text { Median= } \\
12 \text { days } \\
\text { (range= 7-35 } \\
\text { days) } \\
{[\text { Mean }=} \\
13( \pm 8) \\
\text { days }]\end{array}$ & NA & NA \\
\hline 2 & $\begin{array}{l}\text { Kirchner H. } \\
\text { (2011)[19] }\end{array}$ & 20 & $\begin{array}{l}15 \text { patients }= \\
1 \\
5 \text { patients }=2\end{array}$ & $\begin{array}{l}15 \text { patients }= \\
7.96 \text { (range }= \\
3.83-64.58) \\
5 \text { patients }= \\
\text { range }=2.72 \text { to } \\
13.65\end{array}$ & NA & $\begin{array}{l}\text { Median= } \\
11 \text { days } \\
\text { (range }= \\
9-19 \text { days) }\end{array}$ & NA & NA & $\begin{array}{l}\text { Bone pain } \\
\text { during regen- } \\
\text { eration phase }\end{array}$ \\
\hline 3 & $\begin{array}{l}\text { Sammassimo } \\
\text { S. et al. } \\
\text { (2011)[20] }\end{array}$ & 15 & NA & NA & NA & $\begin{array}{l}\text { Median= } \\
11 \text { days } \\
\text { (range= } \\
7-13 \text { ) }\end{array}$ & $\begin{array}{l}\text { Median= } \\
12 \text { days } \\
\text { (range= } \\
7-22)\end{array}$ & NA & $\begin{array}{l}\text { 9/15- } \\
\text { Febrile neutro- } \\
\text { penia }\end{array}$ \\
\hline 4 & $\begin{array}{l}\text { Sever M. et al. } \\
\text { (2012)[21] }\end{array}$ & 24 & NA & NA & $\begin{array}{l}\text { Median= } \\
28.49 \text { (range= } \\
7.5-167.72)\end{array}$ & NA & NA & NA & $\begin{array}{l}4 / 24- \\
\text { Mild muscle } \\
\text { and bone pain }\end{array}$ \\
\hline 5 & $\begin{array}{l}\text { Andreola G. } \\
\text { et al. } \\
\text { (2012)[22] }\end{array}$ & 14 & $\begin{array}{l}75 \% \text { patients }=1 \\
25 \% \text { patients }=2\end{array}$ & $\begin{array}{l}5.2 \\
\text { (range }= \\
2.2-10.6 \text { ) }\end{array}$ & $\begin{array}{l}\text { Day 4: Medi- } \\
\text { an=16 (range= } \\
3-42 \text { ) }\end{array}$ & $\begin{array}{l}\text { Median= } \\
12 \text { days } \\
\text { (range }=9\end{array}$ & $\begin{array}{l}\text { Median= } \\
13 \text { days } \\
\text { (range }=9\end{array}$ & NA & Bone pain \\
\hline
\end{tabular}




\begin{tabular}{|c|c|c|c|c|c|c|c|c|c|}
\hline & \multirow[t]{3}{*}{ References } & \multirow{3}{*}{$\begin{array}{l}\text { No. of } \\
\text { pa- } \\
\text { tients }\end{array}$} & \multirow{3}{*}{$\begin{array}{l}\text { No. of } \\
\text { apheresis }\end{array}$} & \multirow{3}{*}{$\begin{array}{l}\text { CD34+ cell count } \\
\text { ( X 10\%/kg body } \\
\text { weight) }\end{array}$} & \multirow{3}{*}{$\begin{array}{l}\text { No. of CD34+ } \\
\text { cells/ } / \mu 1\end{array}$} & \multicolumn{3}{|c|}{ Engraftment (Time till regeneration) } & \multirow[t]{3}{*}{ Side Effects } \\
\hline & & & & & & \multirow{2}{*}{\begin{tabular}{|l|}
$\begin{array}{l}\text { Neutro- } \\
\text { phils }\end{array}$ \\
$>0.5 \mathrm{G} / \mathrm{L}$ \\
\end{tabular}} & \multicolumn{2}{|c|}{ Platelets } & \\
\hline & & & & & & & $>20 \mathrm{G} / \mathrm{L}$ & $>50 \mathrm{G} / \mathrm{L}$ & \\
\hline & & & & & $\begin{array}{l}\text { Day 5: } \\
\text { Median= } 60 \\
(\text { range= } 14 \\
-138)^{*}\end{array}$ & -13 days) & -19 days) & & \\
\hline 6 & $\begin{array}{l}\text { Lanza F. et al. } \\
(2012)[23]\end{array}$ & 211 & NA & $\begin{array}{l}68 \% \text { patients }= \\
\geq 2.0\end{array}$ & $\begin{array}{l}83 \% \text { patients } \\
\geq 20 *\end{array}$ & NA & NA & NA & NA \\
\hline 7 & $\begin{array}{l}\text { Lazlo D. et al. } \\
\text { (2012)[24] }\end{array}$ & 21 & $\begin{array}{l}\text { Median=1 } \\
\text { (range }=1-2 \text { ) }\end{array}$ & $\begin{array}{l}\text { Median= } 4 \\
(\text { range }=2.2-10.6)\end{array}$ & $\begin{array}{l}\text { Day 5: } \\
\text { Median= } 52 \\
(\text { range= } \\
10-138)^{*}\end{array}$ & NA & NA & NA & NA \\
\hline 8 & $\begin{array}{l}\text { Morabito L. } \\
\text { et al. } \\
(2012)[25]\end{array}$ & 4 & $\begin{array}{l}\text { Median=1 } \\
\text { (range }=1-2 \text { ) }\end{array}$ & $\begin{array}{l}\text { Median= } 5.6 \\
\text { (range= } 2.4-8.4 \text { ) }\end{array}$ & $\begin{array}{l}\text { Median= } 91 \\
\text { (range }= \\
13-138)^{*}\end{array}$ & NA & NA & NA & NA \\
\hline 9 & $\begin{array}{l}\text { Czerw T. et al. } \\
\text { (2012)[26] }\end{array}$ & 55 & NA & $6.7 \pm 3$ & NA & $\begin{array}{l}\text { Median= } \\
12 \text { days } \\
\text { (range= } \\
10-13 \text { ) }\end{array}$ & NA & $\begin{array}{l}\text { Median= } \\
13 \\
(\text { range= } \\
0-19)\end{array}$ & $\begin{array}{l}\text { 9/55- } \\
\text { Grade } 3 \text { or } 4 \\
\text { Infection } \\
\text { 3/55-Neutrope } \\
\text { nic fever }\end{array}$ \\
\hline 10 & $\begin{array}{l}\text { Dmoszynska } \\
\text { A. et al. } \\
\text { (2012)[27] }\end{array}$ & 54 & 1 & $\begin{array}{l}\text { Median= 9.1 } \\
(\text { range }=0-23)\end{array}$ & $\begin{array}{l}\text { Median= } 62.0 \\
(\text { range }= \\
2-394)\end{array}$ & NA & NA & NA & $\begin{array}{l}\text { 9/54- Neutro- } \\
\text { penic fever } \\
8 / 54- \\
\text { Bone pain }\end{array}$ \\
\hline 11 & $\begin{array}{l}\text { Yafour N. et } \\
\text { al. (2013)[28] }\end{array}$ & 10 & $\begin{array}{l}\text { Median=1 } \\
(\text { range }= \\
1-2)\end{array}$ & $\begin{array}{l}\text { Median= } 4.09 \\
\text { (range }=0.25- \\
4.84)\end{array}$ & NA & NA & NA & NA & $\begin{array}{l}\text { 3/10- Bone pain } \\
\text { 3/10- Headache }\end{array}$ \\
\hline 12 & $\begin{array}{l}\text { Kotwica K. } \\
\text { et al. } \\
\text { (2012)[29] }\end{array}$ & 23 & NA & $\begin{array}{l}\text { Mean } \pm S D= \\
10.1 \pm 4.0\end{array}$ & NA & $\begin{array}{l}\text { Mean } \pm \\
\mathrm{SD}= \\
13.0 \pm 4.0 \\
\text { days }\end{array}$ & $\begin{array}{l}\text { Mean } \pm \\
\mathrm{SD}= \\
16.1 \pm 4.4 \\
\text { days }\end{array}$ & NA & $\begin{array}{l}\text { 4/23- Neutro- } \\
\text { penic fever } \\
1 / 23 \text { - Neutro- } \\
\text { penic entero- } \\
\text { colitis } \\
1 / 23 \text { - Sepsis }\end{array}$ \\
\hline 13 & $\begin{array}{l}\text { Gopcsa L. } \\
\text { et al. } \\
\text { (2013)[30] }\end{array}$ & 21 & 1 & $\begin{array}{l}\begin{array}{l}\text { Median=3 } \\
(\text { range }=\end{array} \\
0.81-24.7)\end{array}$ & NA & NA & NA & NA & NA \\
\hline 14 & $\begin{array}{l}\text { Ostuni A. } \\
\text { et al. } \\
\text { (2013)[31] }\end{array}$ & 44 & Mean $=1.45$ & $\begin{array}{l}\text { Median= } \\
4.3 \\
(\text { range }= \\
0.8-6.2)\end{array}$ & $\begin{array}{l}\text { Median= } \\
58.3 \\
(\text { range= } \\
10- \\
503.5 \text { ) }\end{array}$ & $\begin{array}{l}\text { Median= } \\
12 \text { days } \\
\text { (range= } \\
10-23)\end{array}$ & $\begin{array}{l}\text { Median= } \\
14 \text { days } \\
\text { (range= } \\
10-33 \text { ) }\end{array}$ & NA & NA \\
\hline 15 & $\begin{array}{l}\text { Sever M. } \\
\text { et al. } \\
\text { (2013)[32] }\end{array}$ & 16 & NA & NA & $\begin{array}{l}\text { Median= } \\
32 \\
\text { (range= } \\
16.94- \\
189.76)\end{array}$ & NA & NA & NA & NA \\
\hline 16 & $\begin{array}{l}\text { De Giorgi U. } \\
\text { et al. } \\
\text { (2012)[33] }\end{array}$ & 22 & NA & NA & NA & $\begin{array}{l}\text { Median= } \\
15 \text { days }\end{array}$ & NA & NA & NA \\
\hline 17 & $\begin{array}{l}\text { Lefrere F. et.al. } \\
\text { (2011)[34] }\end{array}$ & 40 & $\begin{array}{l}\text { Median=1 } \\
(\text { range }=1-3)\end{array}$ & $\begin{array}{l}\text { Median= } 5.50 \\
\text { (range= } 1.1-20)\end{array}$ & $\begin{array}{l}\text { Median= } 55.5 \\
(\text { range }=1-196)\end{array}$ & $\begin{array}{l}\text { Median= } \\
14 \text { days } \\
\text { (range= } \\
9-21)\end{array}$ & $\begin{array}{l}\text { Median= } \\
12 \text { days } \\
\text { (range= } \\
6-19)\end{array}$ & NA & $\begin{array}{l}\text { 14/40- Bone } \\
\text { pain and/or } \\
\text { headache }\end{array}$ \\
\hline
\end{tabular}


Table 4. Mobilization and engraftment data of healthy donors that underwent hematopoietic stem cell mobilization with biosimilar G-CSF for allogeneic stem cell transplantation

\begin{tabular}{|c|c|c|c|c|c|c|c|c|c|}
\hline & References & No. of & No. of apher- & $\mathrm{CD} 4^{+}$cell count & No. of CD34+ & Engraftment & Time till reg & neration) & Side Effects \\
\hline & & donors & esis & ( X 106/kg body & cells/ $\mu \mathrm{l}$ & Neutrophils & Platelets & & \\
\hline & & & & & & $>0.5 \mathrm{G} / \mathrm{L}$ & $>20 \mathrm{G} / \mathrm{L}$ & $>50 \mathrm{G} / \mathrm{L}$ & \\
\hline 1 & $\begin{array}{l}\text { Schmitt M. } \\
\text { et al. (2013)[35] }\end{array}$ & 11 & 1.45 & $\begin{array}{l}\text { Median= } 4.4 \\
(\text { range }=2.0-7.3)\end{array}$ & $\begin{array}{l}\text { Median= } 65.8 \\
(\text { range }= \\
19.3 \text { to 114.6) }\end{array}$ & $\begin{array}{l}\begin{array}{l}\text { Median }=14 \\
\text { days } \\
\text { (range= } \\
11-20 \text { days) }\end{array}\end{array}$ & $\begin{array}{l}\text { Median }= \\
6 \text { days } \\
\text { (range }= \\
0-8 \text { days) }\end{array}$ & $\begin{array}{l}\text { Median }= \\
13 \text { days } \\
\text { (range= } \\
11-46 \\
\text { days) }\end{array}$ & $\begin{array}{l}1 / 11 \text { - Flu-like } \\
\text { symptoms } \\
1 / 11 \text { - Back pain }\end{array}$ \\
\hline 2 & $\begin{array}{l}\text { Nagler A. } \\
\text { et al. (un- } \\
\text { published) }\end{array}$ & 22 & $\begin{array}{l}21 \text { donors }=1 \\
1 \text { donor }=2\end{array}$ & $9.44 \pm 4.76$ & $\begin{array}{l}\text { Median }= \\
64 \\
(\text { range }=18-193)\end{array}$ & $\begin{array}{l}\text { Median= } \\
15 \text { days } \\
\text { (range= } \\
11-20)\end{array}$ & $\begin{array}{l}\text { Median= } \\
9 \text { days } \\
\text { (range= } \\
8-14 \text { ) }\end{array}$ & $\begin{array}{l}\text { Median } \\
=12 \text { days } \\
\text { (range= } \\
10-20 \text { ) }\end{array}$ & Bone pain \\
\hline 3 & $\begin{array}{l}\text { Nagler A. } \\
\text { et al. (2013) [36] }\end{array}$ & 24 & $\begin{array}{l}\text { Mean=1.3; } \\
19 \text { donors }=1 \\
4 \text { donors }=2 \\
1 \text { donor }=3\end{array}$ & $\begin{array}{l}10.2 \\
\text { (range }=0.93-35.4)\end{array}$ & NA & $\begin{array}{l}\text { Median= } \\
13 \text { days } \\
\text { (range= } \\
10-21)\end{array}$ & $\begin{array}{l}\text { Median= } \\
16 \text { days } \\
\text { (range= } \\
12-33 \text { ) }\end{array}$ & $\begin{array}{l}\text { Median = } \\
17 \text { days } \\
\text { (range= } \\
12-33 \text { ) }\end{array}$ & $\begin{array}{l}12 / 24=\text { mild } \\
\text { arthralgia; } \\
2 / 24=\text { Flu-like } \\
\text { symptoms }\end{array}$ \\
\hline 4 & $\begin{array}{l}\text { Antelo ML. } \\
\text { et al. (2013)[37] }\end{array}$ & 9 & $\begin{array}{l}\text { Median=1 } \\
\text { (range= } \\
1-2)\end{array}$ & $\begin{array}{l}\text { Median= } \\
7.2 \\
\text { (range= } \\
4-9.2 \text { ) }\end{array}$ & $\begin{array}{l}\text { Median= } \\
70.2 \times 10^{9} / \mathrm{L} \\
(\text { range= } \\
24-114)\end{array}$ & NA & 25 days & NA & $\begin{array}{l}\text { 9/9- Mild bone/ } \\
\text { muscle pain }\end{array}$ \\
\hline 5 & $\begin{array}{l}\text { Becker PSA. } \\
\text { et al. (2013)[38] }\end{array}$ & 69 & $\begin{array}{l}93 \% \text { donors }= \\
1 \\
7 \% \text { donors }=2\end{array}$ & NA & $\begin{array}{l}\text { Mean= } \\
111 / \mathrm{L} \\
\text { (range= } \\
34-284)\end{array}$ & NA & NA & NA & $\begin{array}{l}\text { 62/69-Bone pain } \\
1 / 69 \text { - Chest pain } \\
\text { (SAE) }\end{array}$ \\
\hline 6 & $\begin{array}{l}\text { Azar N. et al. } \\
\text { (2012)[39] }\end{array}$ & 21 & $\begin{array}{l}11 \text { donors }=1 \\
9 \text { donors } \\
=2 \\
1 \text { donor }= \\
3\end{array}$ & $\begin{array}{l}\text { Median }=6.0 \\
(\text { range }=2.6-9.2)\end{array}$ & $\begin{array}{l}\text { Median= } 72 \\
(\text { range }= \\
16-145)\end{array}$ & NA & NA & NA & 8/21-Bone pain \\
\hline
\end{tabular}

\section{The global use of biosimilar G-CSF for mobilization of progenitor cells in autologous and allogeneic stem cell transplantation centers}

We have summarized the available data on the use of biosimilar G-CSF for mobilization of hematopoietic stem cells in patients with a range of hematopoietic malignancies for use in subsequent autologous stem cell transplantation and the mobilization in related and volunteer unrelated normal donors. Biosimilar G-CSF was used for mobilization in more than 900 individuals including patients with hematological malignancies, germ cell tumors and cardiac failure and normal family related (sibling) as well as unrelated volunteer donors from donor registries. Biosimilar G-CSF was found to be safe with limited and transient toxicity, good mobilization yield and transplantation outcomes equivalent to the originator filgrastim (Neupogen ${ }^{\circledR}$ ).

Based on the available data in 904 individuals we may conclude that biosimilar G-CSF can be used to mobilize peripheral blood hematopoietic stem cells with equivalent efficiency to that of the originator G-CSF. No significant differences have been demonstrated between biosimilar G-CSF and Neupogen ${ }^{\circledR}$ for the key parameters measured for PBSCs harvest and for engraftment post transplantation as well as to the frequency of occurrence of side effects in donors of both autologous and allogeneic stem cells.

Importantly, published reports have indicated that there was no increase in toxicity or development of side effects either at the time of the mobilization or during follow-up (although still relatively short) using biosimilar G-CSF rather than the originator Filgrastim G-CSF. Pharmacovigilance data for Ratiograstim ${ }^{\circledR} /$ Tevagrastim ${ }^{\circledR}$ are now based on more than 100,000 patients (data on file) who received XM02 because of neutropenia after chemotherapy for a solid tumor, leukemia or lymphoma.[21, 22, 24, 40-46] All three biosimilar G-CSF products currently licensed in the European Union (EU) have similar safety profiles and were equal to originator G-CSF.[47] The increasing body of data summarizing the experience of using biosimilar G-CSF in patients with chemotherapy induced neutropenia and for the mobilization of autologous PBSC shows that biosimi- 
lar G-CSF is safe and as effective in PBSC mobilization as the originator G-CSF. In addition, there is a small but significant and growing experience in the use of biosimilar G-CSF in the successful and safe mobilization of PBSC from matched related and unrelated volunteer donors.

\section{Future directions}

The possibility for functional differences between biosimilars and their originator products has led to the development of specific guidelines by the European Medicines Agency (EMA) specifying the minimum requirements for the approval of biosimilars. In the guidelines, the basic premise is that biosimilars must demonstrate comparable efficacy and safety to the originator product. The guidelines require evidences related to the pre-clinical pharmacodynamics and toxicity, clinical pharmacokinetic and pharmacodynamic data and clinical efficacy (phase III) studies. The EMA recommended that if the efficacy of G-CSF is demonstrated in the setting of chemotherapy induced neutropenia, then extrapolation to other indications is allowed. Clinical safety data should be collected for a minimum of six months and the importance of ongoing pharmacovigilance is also stressed.

\section{Health economy aspects}

Use of a biosimilar G-CSF has significant cost advantages for a transplant unit.[18] When first introduced in 2008, biosimilars were approximately $15 \%$ cheaper than the originator. Today the price of biosimilars has been reduced rapidly, up to $80 \%$ lower in cost.[18] Thus, the use of biosimilar G-CSF is cost-effective strategy permitting a reduction of cost compared with the use of originator G-CSF. The savings can be re-invested in other services.[48]

An important concern frequently raised regarding the use of biosimilars is the potential for immunogenicity. Any protein used as a drug has the potential to cause immunogenicity. In a thorough literature search we performed we found only very limited data relating to immunogenicity for any type of G-CSF.[16] In this regard, there are some published data suggesting that G-CSF is non-immunogenic.[49] Conversely, anti-G-CSF antibodies were found in 15/135 healthy individuals who had never been exposed to G-CSF.[50] Data submitted to the EMA found no significant difference between biosimilar G-CSF (Nivestim ${ }^{\circledR}$, Hospira) and the reference G-CSF Neupogen ${ }^{\circledR}$ in terms of immunogenicity. Continuous long term follow-ups in very large cohorts of healthy donors are obviously needed for the evaluation of immunogenicity, a still rare but potentially important side effect to be ruled out.

\section{Conclusions}

This report summarizes the currently available experience using biosimilar G-CSF for stem cell mobilization and may help to dispel some of the concerns appropriately raised regarding their use in this clinical setting,[4-8] especially in healthy related donors and unrelated volunteer donors. As healthy donors gain no personal benefit from the procedure, absolute assurance of no harm to them is mandatory. Correctly detailing these concerns a recent review regarding the use of biosimilar G-CSF, in healthy donors, suggested that safety data can only be obtained by performing an adequate number of stem cell mobilization procedures and conducting long-term follow up in patients undergoing allogeneic stem cell transplantation.[16] The parameters to be analyzed were not specified. Currently both the WMDA and EBMT do not advise the use of a biosimilar G-CSF in the healthy donor setting unless in the context of clinical trials where both patients and donors give informed consent. This review analyses in retrospective more than 900 patients and healthy donors that have been mobilized with biosimilar G-CSF for both autologous and allogeneic transplantations and to our knowledge all data published at present have shown equivalence for biosimilar G-CSF when used for stem cell mobilization. Our study is not a randomized study but a summary of data from various institutions possibly involving different protocols including the use of variable doses of originator/biosimilar G-CSF. Biosimilar G-CSF products were approved by the EMA in 2008 and have been licensed only in the last few years. Therefore long-term safety for both donor and recipient of the graft can be only evaluated over the forthcoming years. Indeed, long-term follow-up data will be collected in ongoing studies.

In summary, we present the published experience for the use of biosimilar G-CSFs in more than 900 patients and normal family related and volunteer unrelated donors. The toxicity profile, PBSC yield and efficacy seem equivalent to historical data with the reference G-CSF filgrastim. Until results from multi-center randomized clinical trials that directly compare biosimilar G-CSF with the originator G-CSF are reported, it is important to collect and summarize all of the available clinical experience in order to allow the transplant community to make informed decisions regarding the choice of G-CSF.

\section{Competing Interests}

AS, MS, AP, KHO, AN received travel grants. $\mathrm{AN}, \mathrm{KHO}$ and MS received research grants and speaker's honoraria from TEVA. MG received speaker's and consultant's honoraria from TEVA. AP re- 
ceived an unrestricted educational grant from TEVA Ltd. MS received speaker's honoraria from AMGEN Ltd. JM, LW, PT and RK declare no conflict of interest.

\section{References}

1. Aapro MS, Cameron DA, Pettengell R, Bohlius J, Crawford J, Ellis M, et al. EORTC guidelines for the use of granulocyte-colony stimulating factor to reduce the incidence of chemotherapy-induced febrile neutropenia in adult patients with lymphomas and solid tumours. Eur J Cancer. 2006; 42: 2433-53.

2. Smith TJ, Khatcheressian J, Lyman GH, Ozer H, Armitage JO, Balducci L, et al. 2006 update of recommendations for the use of white blood cell growth factors: an evidence-based clinical practice guideline. J Clin Oncol. 2006; 24: 3187-205

3. [Internet] European Medicines Agency. http://www.ema.europa.eu/docs/ en GB/document library/Scientific guideline/2009/09/WC500003955.pdf

4. Niederwieser D, Schmitz S. Biosimilar agents in oncology/haematology: from approval to practice. Eur J Haematol. 2011; 86: 277-88.

5. Gascon P. Presently available biosimilars in hematology-oncology: G-CSF. Target Oncol. 2012; 7 (Suppl 1): S29-S34.

6. Schofield I. EC defends biosimilar safety. Scrip. 2008; 1: 3358.

7. Mellstedt $\mathrm{H}$, Niederwieser D, Ludwig H. The challenge of biosimilars. Ann Oncol. 2008; 19: 411-9.

8. Weise M, Bielsky MC, De Smet K, Ehmann F, Ekman N, Giezen TJ, et al. Biosimilars: what clinicians should know. Blood. 2012; 120: 5111-7.

9. [Internet] European Medicines Agency. http://www.ema.europa.eu/docs/ en_GB/document_library/EPAR_-_Public_assessment_report/human/00082 5/WC500047793.pdf

10. Engert A, del Giglio A, Bias P, Lubenau H, Gatzemeier U, Heigener D. Incidence of febrile neutropenia and myelotoxicity of chemotherapy: a meta-analysis of biosimilar G-CSF studies in breast cancer, lung cancer, and non-Hodgkin's lymphoma. Onkologie. 2009; 32: 599-604.

11. Sheridan B, Fox A. Feature on biopharmaceuticals: Views from the pharmaceutical industry - significant questions relating to efficacy and immunogenicity will remain at approval. Eur J Hosp Pharm Pract. 2007; 13: 70-3.

12. [Internet] World Health Organization. http://www.who.int/biologicals/ areas/biological_therapeutics/BIOTHERAPEUTICS_FOR_WEB_22APRIL201 $0 . p d f$

13. Koradi R, Billeter M, Wuthrich K. MOLMOL: a program for display and analysis of macromolecular structures. J Mol Graph. 1996; 14: 51-5, 29-32.

14. Pettersen EF, Goddard TD, Huang CC, Couch GS, Greenblatt DM, Meng EC, et al. UCSF Chimera--a visualization system for exploratory research and analysis. J Comput Chem. 2004; 25: 1605-12.

15. Zink T, Ross A, Luers K, Cieslar C, Rudolph R, Holak TA. Structure and dynamics of the human granulocyte colony-stimulating factor determined by NMR spectroscopy. Loop mobility in a four-helix-bundle protein. Biochemistry. 1994; 33: 8453-63.

16. Shaw BE, Confer DL, Hwang WY, Pamphilon DH, Pulsipher MA. Concerns about the use of biosimilar granulocyte colony-stimulating factors for the mobilization of stem cells in normal donors: position of the World Marrow Donor Association. Haematologica 2011; 96: 942-7.

17. [Internet] European Group for Blood and Marrow Transplantation. Niederwieser D. http://www.worldmarrow.org/fileadmin/Committees/ CLWG/Biosimilars/Biosimilars_9Jan09.pdf

18. Publicover A, Richardson DS, Davies A, Hill KS, Hurlock C, Hutchins D, et al. Use of a biosimilar granulocyte colony-stimulating factor for peripheral blood stem cell mobilization: an analysis of mobilization and engraftment. Brit J Haematol. 2013; 162: 107-11.

19. Kirchner H. Mobilisierung von stammzellen mit Ratiograstim $₫$ im autologen setting. Deutschland: Wolters Kluwer Health Pharma Solutions 2011.

20. Sammassimo S, Andreola G, Bassi S, Paochi P, Gardellini A, Minga P, et al. Biosimilar G-CSF in post autologous stem cell transplantation setting: preliminary results. Haematologica. 2011; 96 (Suppl 2): S589. http://www.haematologica.org/content/96/supplement_2/1.full.pdf

21. Sever M, Domanovic D, Vrtovec B, Lezaic L, Pretnar J, Poglajen G, et al. Mobilisation of heart failure patients using biosimilar granulocyte colony stimulating factor (TevaGrastim) for autologous CD34+ cells preparation and application into the heart. Bone Marrow Transplant. 2012; 47: S243. http://www.nature.com/bmt/journal/v47/n1s/pdf/bmt201237a.pdf

22. Andreola $G$, Babic A, Rabascio C, Negri M, Martinelli G, Laszlo D. Plerixafor and Filgrastim XM02 (Tevagastrim) as a first line peripheral blood stem cell mobilisation strategy in patients with multiple myeloma and lymphoma candidated to autologous bone marrow transplantation. Eur J Haematol. 2012; 88: $154-8$

23. Lanza F, Laszlo D, Pasini A, Guerrasio A, Martino M, Milone G, et al. Plerixafor pus filgrastim biosimilar can mobilise CD34+ progenitor cells from multiple myeloma and lymphoma patients failing previous mobilisation attempts: an Italian multicentre study. Bone Marrow Transplant. 2012; 47: S239. http://www.nature.com/bmt/journal/v47/n1s/pdf/bmt201237a.pdf

24. Laszlo D, Andreola G, Babic A, Rabascio C, Montinaro A, Morabito L, et al. Plerixafor in combination with originator or biosimilar XM02-G-CSF as first-line peripheral blood stem cell mobilisation strategy in patients with lymphomas and multiple myeloma candidate to ASCT: a single-centre experience. Bone Marrow Transplant. 2012; 47: S236-S7. http://www.nature.com/bmt/journal/v47/n1s/pdf/bmt201237a.pdf

25. Morabito L, Andreola G, Babic A, Montinaro A, Elezi R, Rabascio C, et al. Plerixafor and G-CSF combination leads to excellent PBSC mobilisation and collection in Jehovah's Witnesses patients: experience at the European Institute of Oncology. Bone Marrow Transplant. 2012; 47: S236 http://www.nature.com/bmt/journal/v47/n1s/pdf/bmt201237a.pdf

26. Czerw T, Kruzel T, Sadus-Wojciechowska M, Najda J, Holowiecki J, Giebel S. Comparison of two formulations of filgrastim, Neupogen (Amgen) and Zarzio (Sandoz), used to accelerate neutrophil recovery after autologous peripheral blood stem cell transplantation. Bone Marrow Transplant. 2012; 47: S316. http://www.nature.com/bmt/journal/v47/n1s/pdf/bmt201237a.pdf

27. Dmoszynska A, Manko J, Croneck A, Grzasko N, Klimek P, Nowaczynska A, et al. Comparable efficacy and safety of biosimilar and originator G-CSF in patients undergoing haematopoietic stem cell mobilisation. Bone Marrow Transplant. 2012; 47: S231 http://www.nature.com/bmt/journal/v47/n1s/ pdf/bmt201237a.pdf

28. Yafour N, Brahimi M, Osmani S, Arabi A, Bouhass R, Bekadja MA. Biosimilar G-CSF (filgrastim) is effective for peripheral blood stem cell mobilization and non-cryopreserved autologous transplantation. Transfus Clin Biol. 2013; doi:10.1016/j.tracli.2013.04.109.

29. Kotwica K, Cioch M, Wach M, Manko J, Jawniak D, Croneck A, et al. Biosimilar G-CSF is effective in reducing the duration of neutropenia after autologous bone marrow transplantation. Bone Marrow Transplant. 2012; 47: S316 http://www.nature.com/bmt/journal/v47/n1s/pdf/bmt201237a.pdf

30. Gopcsa L, Remenyi P, Adamkovich N, Barta A, Batai A, Farkas Z, et al. Experiences of autologous peripheral blood stem cell mobilization and engraftment after autologous stem cell transplantation with "biosimilar" filgrastim (Zarzio®,Sandoz, granulocyte colony-stimulating factor). Bone Marrow Transplant. 2013; 48: S114. http://registration.akm.ch/einsicht.php? XNABSTRACT ID $=170146 \&$ XNSPRACHE ID $=2 \&$ XNKONGRESS ID $=179 \&$ XNMASKEN_ID $=900$

31. Ostuni A, Morciano MR, Mele A, Fina M, Carlino D, Greco G, et al. Zarzio plus chemotherapy as peripheral blood stem cell mobilization strategy in patients with haematological diseases candidated to autologous stem cell transplantation. Bone Marrow Transplant. 2013; 48: S120.

32. Sever $M$, Vrtovec B, Cukjati $M$, Lezaic L, Poglajen $G$, Socan A, et al. Mobliization using biosimilar granulocyte colony stimulating factor (Zarzio) for autologous CD34+ cells preparation and application into the heart of patients with dilative cardiomyopathy. Bone Marrow Transplant. 2013; 48: S192. http://registration.akm.ch/einsicht.php?XNABSTRACT_ID $=170424 \& X$ NSPRACHE_ID $=2 \&$ XNKONGRESS_ID $=179 \& X N M A S K E N \_I D=900$

33. De Giorgi U, Rosti G, Papiani G, Kopf B, De Vivo R, Gentile A, et al. Multi-cycle high-dose chemotherapy with ti-ce regimen for relapsed/refractory patients with germ cell tumours: the Romagna cancer institute experience. Bone Marrow Transplant. 2012; 47: S426. http://www.nature.com/bmt/journal/v47/n1s/pdf/bmt201237a.pdf

34. Lefrere F, Brignier AC, Elie C, Ribeil JA, Bernimoulin M, Aoun C, et al. First experience of autologous peripheral blood stem cell mobilization with biosimilar granulocyte colony-stimulating factor. Adv Ther. 2011; 28: 304-10.

35. Schmitt $\mathrm{M}, \mathrm{Xu}$ X, Hilgendorf I, Schneider C, Borchert $\mathrm{K}$, Gläser D, et al. Mobilization of PBSC for allogeneic transplantation by the use of the G-CSF biosimilar XM02 in healthy donors. Bone Marrow Transplant 2013; 48:922-5.

36. Danylesko I, Rina S, Bloom-Varda N, Yerushalmi R, Shem-Tov N, Volchek Y, et al. The Use Of Tevagrastim (Biosimilar Filgrastim XMO2) For Hematopoietic Stem Cell Mobilization In HLA Matched Sibling Donors For Allogeneic Stem Cell Transplantation To AML/MDS Patients. Annual Meeting of ASH. 2013. https://ash.confex.com/ash/2013/webprogram/ Paper59988.html

37. Antelo ML, Zabalza A, Sancilez Anton MP, Zalba S, Aznar M, Vicente E, et al. Safety and efficacy of a G-CSF biosimilar (Zarzio $($ ) ) for haematopoietic progenitor cell mobilization in allogeneic healthy donors. Bone Marrow Transplant. 2013; 48: S102. http://registration.akm.ch/einsicht.php? XNABSTRACT_ID $=169532 \& X N S P R A C H E I D=2 \& X N K O N G R E S S$ ID $=179 \&$ XNMASKEN_ID $=900$

38. Becker PSA, Brauninger S, Bialleck H, Luxembourg B, Schulz M, Wiesneth M, et al. Biosimilar filgrastim mobilizes haematopoietic stem cells in healthy volunteer donors with expected efficiency and typical acute adverse effects:interim results of a post authorization safety study. Bone Marrow Transplant. 2013; 48: S28. http://registration.akm.ch/einsicht.php? XNABSTRACT ID $=161946 \& X N S P R A C H E \_I D=2 \& X N K O N G R E S S I D=179 \&$ XNMASKEN_ID $=900$

39. Azar N, Choquet S, Garnier A, Roos Weil D, Leblond V. Use of a biosimilar G-CSF in allogeneic stem cell mobilisation. Bone Marrow Transplant. 2012; 47: S244. http://www.nature.com/bmt/journal/v47/n1s/pdf/bmt201237a.pdf

40. del Giglio A, Eniu A, Ganae-Motan D, Topuzov E, Lubenau H. XM02 is superior to placebo and equivalent to Neupogen ${ }^{\mathrm{TM}}$ in reducing the duration of severe neutropenia and the incidence of febrile neutropenia in cycle I in breast cancer patients receiving decetaxel/doxorubicin chemotherapy. BCM Cancer. 2008; 8: 332-9.

41. Lubenau H, Sveikata A, Gumbrevicius G, Macijauskiene J, Fokas V, Kazlauskas S, et al. Bioequivalence of two recombinant granulocyte colony-stimulating factor products after subcutaneous injection in healthy volunteers. Int J Clin Pharmacol Ther. 2009; 47: 275-82. 
42. Lubenau H, Bias P, Maly A-K, Siegler KE, Mehltretter K. Pharmakokinetic and pharmacodynamic profile of new biosimilar Filgrastim XM02 equivant to market Filgrastim Neupogen ${ }^{8}$. Biodrugs 2009; 23: 43-51.

43. Gatzemeier U, Ciuleanu T, Dediu M, Ganea-Motan E, Lubenau H, Del Giglio A. XM02, the first biosimilar G-CSF, is safe and effective in reducing the duration of severe neutropenia and incidence of febrile neutropenia in patients with small cell or non-small cell lung cancer receiving platinum-based chemotherapy. J Thorac Oncol. 2009; 4: 736-40.

44. Engert A, Griskevicius L, Zyuzgin Y, Lubenau H, del Giglio A. XM02, the first granulocyte colony-stimulating factor biosimilar, is safe and effective in reducing the duration of severe neutropenia and incidence of febrile neutropenia in patients with non-Hodgkin lymphoma receiving chemotherapy. Leuk Lymphoma. 2009; 50: 374-9.

45. Schmitt M, Diestel L, Xu X, Borchert K, Gläser D, Hilgendorf I, et al Application of the G-CSF biosimilar Ratiograstim ${ }^{\circledR}$ for he mobilisation of peripheral stem cells in healthy donors. Onkologie. 2011; 34: 280.

46. Publicover A, Richardson DS, Hill K, Hurlock C, Casey P, Newman J, et al. Use of biosimilar GCSF for peripheral blood progenitor cell mobilisation prior to autologous stem cell transplantation: a single-centre experience. Bone Marrow Transplant. 2010; 45: S158. http://www.nature.com/bmt/journal/ v45/n2s/pdf/bmt201041a.pdf

47. Abraham I, Tharmarajah S, MacDonald K. Clinical safety of biosimilar recombinant human granulocyte colony-stimulating factors. Expert Opin Drug Saf. 2013; 12: 235-46.

48. Aapro M, Cornes P, Abraham I. Comparative cost-efficiency across the European G5 countries of various regimens of filgrastim, biosimilar filgrastim, and pegfilgrastim to reduce the incidence of chemotherapy-induced febrile neutropenia. J Oncol Pharm Pract. 2012; 18: 171-9.

49. Laricchia-Robbio L, Moscato S, Genua A, Liberati AM, Revoltella RP. Naturally occurring and therapy-induced antibodies to human granulocyte colony-stimulating factor (G-CSF) in human serum. J Cell Physiol. 1997; 173: 219-26.

50. Wadhwa M, Bird C, Dilger P, Gaines-Das R, Thorpe R. Strategies for detection, measurement and characterization of unwanted antibodies induced by therapeutic biologicals. J Immunol Methods. 2003; 278: 1-17. 